\title{
Reducibility, a constructive dual of spatiality ${ }^{1}$
}

\author{
FRANCESCO CIRAULO
}

GIOVANNI SAMBIN

\begin{abstract}
An intuitionistic analysis of the relationship between pointfree and pointwise topology brings new notions to light that are hidden from a classical viewpoint. In this paper, we study one of these, namely the notion of reducibility for a pointfree topology, which is classically equivalent to spatiality. We study its basic properties and we relate it to spatiality and to other concepts in constructive topology. We also analyse some notable examples. For instance, reducibility for the pointfree Cantor space amounts to a strong version of Weak König's Lemma.
\end{abstract}

2010 Mathematics Subject Classification 54A05 (primary); 03F55, 54H12, 06D22 (secondary)

Keywords: pointfree topology, spatiality, constructive mathematics

\section{Introduction}

A characteristic feature of constructive Mathematics, that is, Mathematics developed without using the Law of Excluded Middle (LEM), is that each "classical" notion splits into several, no longer equivalent concepts. Even more distinctions appear when also other "usual" foundational principles are omitted, such as the Power-Set Axiom (PSA).

A constructive investigation of the relationships between pointwise and pointfree topology brings new notions to light that were hidden from a classical viewpoint. Reducibility is one of these. It has its origin in the work of the second author [22], and it has already been studied extensively in the case of the Zariski spectrum of a commutative ring by Rinaldi, Sambin and Schuster [19]. Roughly speaking, reducibility is one possible manifestation of a well-known classical notion, that of spatiality for a pointfree topology (locale). Constructively, "spatiality" as in the standard formulation and "reducibility" are two different concepts.

\footnotetext{
${ }^{1}$ This paper is included in the Proceedings of the Fifth Workshop on Formal Topology, Institut Mittag-Leffler, June 2015 (editors Thierry Coquand, Maria Emilia Maietti and Erik Palmgren).
} 
The aim of this paper is to give an introduction to reducibility and prove some of basic results about it. We relate reducibility to other central concepts, first of all spatiality, and we analyse some notable examples. For instance, the pointfree version of the Cantor space reducibility amounts to a version of Weak König's Lemma (WKL). This is in accordance with the well-know fact that spatiality for such a space is Brouwer's Fan Theorem (FAN) and that, classically, FAN and WKL are equivalent.

The notion of a reducible topology was originally introduced in [22] in the framework of so called positive topologies (formal topologies with positivity relations), where it naturally arises. Therefore, after having analysed reducibility in the case of locales, in the second part of the paper we move to the more general framework of positive topologies.

This paper is written in the spirit of a minimalist approach to the foundations of mathematics as developed by Maietti and Sambin [17, 15], though not explicitly within the formal system proposed therein. This essentially means that all definitions and proofs we give remain sound within virtually all possible foundations (usual set theories, topos-valid mathematics, intuitionistic type theories, constructive set theories, et cetera). To achieve this aim, we refrain from using any logical or set-theoretic principle which is not part of even one of the above mentioned foundations. Surely among those principles are LEM and PSA. So the word "constructive" in the title refers to the fact that (i) we use intuitionistic rather than classical logic, and (ii) we take care of distinguishing between arbitrary collections of objects and sets. ${ }^{2}$ A clear advantage of our approach is that all readers can follow our arguments, regardless of their own foundational attitude. From time to time we will remark what would happen if some of these principles were accepted. We will say that a result is classical if it depends on LEM and impredicative if it depends on PSA.

Finally we introduce some notation: for $S$ a set, we write $\mathcal{P}(S)$ for the collection of all subsets of $S$; and for given $U$ and $V$ in $\mathcal{P}(S)$, we write $U \gamma V$ to mean that $U \cap V$ is inhabited.

\section{Preliminaries}

Concrete spaces. Let $(X, \tau)$ be a topological space and let $\mathcal{B}=\{$ ext $a \mid a \in S\}$ be

\footnotetext{
${ }^{2}$ The precise meaning of these two terms depends on the specific foundational theory one adopts. What we have in mind is the Minimalist Foundation of Maietti and Sambin, but other interpretations are possible: for instance, one could read a collection as a (not necessarily proper) class in the usual set-theoretic sense.
} 
a base for $\tau$ indexed by some set $S$. In this case, $(X, \tau)$ can be presented as a triple $\mathcal{X}=(X, \Vdash, S)$ where $x \Vdash a$ is $x \in \operatorname{ext} a$ (plus those conditions on $\Vdash$ which make the ext $a$ 's a base for $\tau$ ). This we call a concrete space: it is a topological space in which the topology is set-based, that is, there exists a base which is a set, and the points form a set.

There are important examples of topologies whose points cannot be assumed to form a set in the minimalist foundation. This happens, for instance, for real numbers defined as Dedekind cuts. On the other hand, the open intervals with rational endpoints, which are indexed by the set $\mathbb{Q} \times \mathbb{Q}$, form a base for such a topology. In fact, there are many concrete examples of topologies like that: a base exists which is a set, even though the whole collection of opens can be too large to be a set predicatively. Thereby, according to Maietti and Sambin [18], our choice of developing topology over a minimalist framework necessarily leads us to a point-free approach, that is, one which is focused on opens rather than on points.

\subsection{Locales and formal topologies}

It is well known that the open sets of a topological space form a frame: they are closed under finite meets and arbitrary joins with respect to inclusion, and binary meets distribute over joins. A frame homomorphism is a map which preserves finite meets and arbitrary joins. Every continuous map between two topological spaces defines, by inverse image, a frame homomorphism between the corresponding frames of opens, but in the opposite direction. A locale is a frame regarded as a generalized topological space. The category of locales is the opposite of the category of frames, so that the direction of arrows coincides with that between topological spaces.

Formal topology is, to a first approximation, a theory of "locales-with-bases", which is suitable for a predicative framework. A predicative approach requires, first of all, a more careful definition of a frame: it is a partially ordered collection with finite meets and set-indexed joins. A base for a frame $L$ is a set $S \subseteq L$ such that $\{a \in S \mid a \leq x\}$ is a set for every $x \in L$, and $x=\bigvee\{a \in S \mid a \leq x\}$. This we call a set-based frame. All the information about a set-based frame can be encoded in a "cover relation", say $\triangleleft$, where $a \triangleleft U$ means $a \leq \bigvee U$, for $a \in S$ and $U \subseteq S$. This is the motivating example for the following definition.

Definition 1.1 A formal cover on a set $S$ is a relation $\triangleleft \subseteq S \times \mathcal{P}(S)$ which satisfies the following conditions for all $a \in S$ and $U, V \subseteq S$ : 
(1) if $a \in U$, then $a \triangleleft U$;

(2) if $a \triangleleft U$ and $u \triangleleft V$ for all $u \in U$, then $a \triangleleft V$; and

(3) if $a \triangleleft U$ and $a \triangleleft V$, then $a \triangleleft \downarrow U \cap \downarrow V$,

where $\downarrow U=\{a \in S \mid a \triangleleft\{u\}$ for some $u \in U\}$.

Each formal cover has an associated frame of formal opens, namely those subsets of the form $\{a \in S \mid a \triangleleft U\}$ for $U \subseteq S$. The set(-indexed family) of all $\downarrow a$ for $a \in S$ is a base for that frame, because every formal open $\{a \in S \mid a \triangleleft U\}$ is the join of all $\downarrow u$ for $u \in U$. So set-based frames are essentially the same thing as formal covers, and a frame homomorphism between two set-based frames can be presented as a(n equivalence class of ) particular binary relations between the corresponding bases (see Sambin [21] for details).

Subobjects. A nucleus on a frame $X$ is a map $j: X \rightarrow X$ such that the conditions $x \leq j(x)=j(j(x))$ and $j(x \wedge y)=j(x) \wedge j(y)$ hold identically. The collection $X_{j}$ of all fixed points of $j$ is a frame, actually a quotient of $X$. As a locale, it corresponds to a regular subobject of $X$; this is called a sublocale.

When a locale is represented as a formal cover $(S, \triangleleft)$, each of its sublocales can be presented as a formal cover $\left(S, \triangleleft^{\prime}\right)$, on the same set $S$, such that

(1) $\triangleleft \subseteq \triangleleft^{\prime}$, and

(2) if $a \triangleleft^{\prime} U$ and $a \triangleleft^{\prime} V$, then $a \triangleleft^{\prime}(\downarrow U \cap \downarrow V)$.

$(\text { See, for instance, Vickers [26]. })^{3}$

\subsection{Points and spatiality}

Given a point $x$ in a topological space $(X, \tau)$, the family $\mathcal{N}(x)$ of all open neighbourhoods of $x$ form a completely prime filter in the frame of all open sets $\tau$. Completely prime filters of $\tau$ correspond to frame homomorphism from $\tau$ to $\mathcal{P}(1)$, the power of the singleton set $1=\{0\}$. A point of a locale $L$ is a frame homomorphism from $L$ to $\mathcal{P}(1)$. In fact, $\mathcal{P}(1)$ is the initial object in the category of frames, that is, the terminal object in the category of locales. Note that $\mathcal{P}(1)$ is a set-based frame which corresponds to the formal cover $(1, \in)$, and it is isomorphic to $2=\{0,1\}$ if and only if LEM holds.

\footnotetext{
${ }^{3}$ Note that (i) a sublocale is presented by a larger cover, and (ii) $\downarrow$ is defined in terms of the ambient cover $\triangleleft$.
} 
Proposition 1.2 Let $L$ be the a set-based frame and let $(S, \triangleleft)$ be the corresponding formal cover. There is a bijection between completely prime filters of $L$ and subsets $\alpha \subseteq S$ which satisfy the following conditions for all $a, b \in S$ and $U \subseteq S$ :

(1) $\alpha$ is inhabited;

(2) if $a \triangleleft U$ and $a \in \alpha$, then $U \curlyvee \alpha$; and

(3) if $a \in \alpha$ and $b \in \alpha$, then $c \in \alpha$ for some $c$ with $c \leq a$ and $c \leq b$.

Proof Here is a sketch of the proof. If $F$ is a completely prime filter of $L$, then $\alpha_{F}=S \cap F$ satisfies all the above conditions. Conversely, given $\alpha$ as above, $F_{\alpha}=$ $\{x \in L \mid \alpha \gamma\{a \in S \mid a \leq x\}\}$ is a completely prime filter.

Definition 1.3 A formal point of a formal cover $\mathcal{S}=(S, \triangleleft)$ is a subset $\alpha \subseteq S$ which satisfies the conditions in the previous proposition. We write $\operatorname{Pt}(\mathcal{S})$ for the collection of formal points of $\mathcal{S}$.

Spatial formal covers. In a concrete space $\mathcal{X}=(X, \Vdash, S)$, the set(-indexed family) $\mathcal{B}=\{\operatorname{ext} a \mid a \in S\}$ is a base for the frame of opens; therefore, the frame of opens corresponds to a formal cover on $\mathcal{B}$. Up to isomorphisms, the same frame can be presented as a formal cover on $S$ itself, namely $(S, \triangleleft \mathcal{X})$, where

$$
a \triangleleft \mathcal{X} U \stackrel{\text { def }}{\Longleftrightarrow} \quad \operatorname{ext} a \subseteq \bigcup_{u \in U} \operatorname{ext} u .
$$

As shown by the second author [22], ext becomes in fact a frame isomorphism between the open subsets of $\mathcal{X}$ and the formal opens of $(S, \triangleleft \mathcal{X})$.

Formal covers of the form $(S, \triangleleft \mathcal{X})$ are spatial, in the sense of the following definition.

Definition 1.4 A formal cover $\mathcal{S}=(S, \triangleleft)$ is spatial if $a \triangleleft U$ follows from the assumption that $a \in \alpha \Rightarrow \alpha \gamma U$ for every formal point $\alpha .^{4}$

For, if $\mathcal{X}$ is a concrete space, then every subset of the form $\diamond x=\{a \in S \mid x \Vdash a\}$ is a formal point, and the condition $a \in \diamond x \Rightarrow \diamond x \succ U$ for all $x \in X$ means precisely $a \triangleleft \mathcal{X} U$.

Impredicatively, a formal cover (locale) $\mathcal{S}=(S, \triangleleft)$ is spatial if and only if there exists a concrete space $\mathcal{X}=(X, \Vdash, S)$ such that $\triangleleft=\triangleleft \mathcal{X}$. In fact, $\mathcal{S}$ is spatial precisely when

\footnotetext{
${ }^{4}$ Note that $a \in \alpha \Rightarrow \alpha \gamma U$ follows from $a \triangleleft U$, for every formal point $\alpha$.
} 
$\triangleleft$ is the cover relation induced by the "large" concrete space $(\operatorname{Pt}(\mathcal{S}), \ni, S)$, where $\operatorname{Pt}(\mathcal{S})$ is the collection of formal points of $\mathcal{S}$.

An example: the topology of radical ideals. Given a commutative ring $R$ with unit, let us write $a \triangleleft U$ when $a$ belongs to the radical ideal $\sqrt{U}$ generated by $U$, as done by Rinaldi, Sambin and Schuster [19]. This is a cover relation whose formal open subsets are precisely the radical ideals of $R$. In this case, the last clause in the definition of a formal cover becomes equivalent to the following: if $a \triangleleft U$ and $a \triangleleft V$, then $a \triangleleft U \cdot V$ where $U \cdot V=\{u \cdot v \mid u \in U$ and $v \in V\}$ and $\cdot$ is multiplication in $R$. This rule corresponds to the well known fact that the lattice of radical ideals is distributive. Moreover, such a cover relation is finitary in the sense that $a \triangleleft U$ holds if and only if $a \triangleleft K$ for some finite subset $K \subseteq U$ (see Ciraulo and Sambin [5] for more details).

Classically, the formal points are just the complement of prime (radical) ideals. Therefore, spatiality holds precisely when, for every $a$ outside a radical ideal $\sqrt{U}$, there exists a prime ideal $P \supseteq U$ such that $a \notin P$. In other words, spatiality means that the radical ideal generated by a given $U \subseteq R$ is the intersection of all prime ideals that contain $U$.

\section{Reducibility for formal topologies and locales}

A locale $L$ is spatial when $x \leq y$ follows from the assumption that $p(x)=1 \Rightarrow p(y)=1$ for every point $p: L \rightarrow \mathcal{P}(1)$. Classically, this is equivalent to saying that whenever $x \not \leq y$ there exists a point $p$ such that $p(x)=1 \neq p(y)$. The aim of the present paper is to study the constructive properties of the following form of spatiality.

Proposition 2.1 In the presence of LEM, the following are equivalent for a locale $L$ :

(1) $L$ is spatial.

(2) For every join-preserving map $\varphi: L \rightarrow \mathcal{P}(1)$ and every $a \in \varphi^{-1}(1)$, there exists a frame homomorphism $p: L \rightarrow \mathcal{P}(1)$, that is, a point of $L$ such that $a \in p^{-1}(1) \subseteq \varphi^{-1}(1)$.

Proof Classically, every $\varphi$ as above is of the form $x \mapsto \neg(x \leq b)$ for some $b \in L$ (take $b=\bigvee\{x \in L \mid \varphi(x)=\emptyset\}$ ) and so (2) becomes exactly spatiality: if $\neg(a \leq b)$, then there exists a point $p$ such that $p(a)=1$ and $p(b)=0$.

Definition 2.2 We call a locale reducible if it satisfies the property (2) in the previous proposition. 
Intuitionistically, every reducible locale $L$ satisfies the following property:

if $\varphi(a)=1$ and $\varphi(b)=0$ for some suplattice homomorphism $\varphi: L \rightarrow \mathcal{P}(1)$, then there is a frame homomorphism $p: L \rightarrow \mathcal{P}(1)$ with $p(a)=1$ and $p(b)=0$.

A join-preserving map $\varphi: L \rightarrow \mathcal{P}(1)$ carries the same information of the completely prime semi-filter (upward closed subset of $L$ inaccessible by joins) $\varphi^{-1}(1)$. If $L$ is the set-based frame presented by a formal cover $(S, \triangleleft)$, then one can show that completely prime semi-filters, and hence join-preserving maps $\varphi: L \rightarrow \mathcal{P}(1)$, correspond to splitting subsets in the sense of the following definition.

Definition 2.3 Let $(S, \triangleleft)$ be a formal cover. A subset $Z \subseteq S$ is called splitting if

$$
a \triangleleft U \& a \in Z \Longrightarrow U \gamma Z
$$

for every $a \in S$ and $U \subseteq S$.

Note that being splitting is part of the definition of a formal point. Note also that splitting subsets are closed under unions.

Definition 2.4 A formal cover $(S, \triangleleft)$ is reducible if for every splitting subset $Z \subseteq S$ and every $a \in Z$, there exists a formal point $\alpha$ such that $a \in \alpha \subseteq Z$.

In other words, a formal cover is reducible when splitting subsets are precisely the unions of formal points.

\subsection{On the relation between reducibility and spatiality}

A discrete locale is one whose underlying frame is $\mathcal{P}(S)$ for some set $S$. Such a frame corresponds to the formal cover $(S, \in)$. Its sublocales are all of the form $(S, \triangleleft)$ where $a \triangleleft U \& a \triangleleft V \Rightarrow a \triangleleft U \cap V$ for all $a \in S$ and $U, V \subseteq S$.

Proposition 2.5 Every sub-locale of a discrete locale is reducible.

Proof Let $\mathcal{S}=(S, \triangleleft)$ be a sublocale of the discrete locale $\mathcal{P}(S)$, and let $a \in Z$ with $Z$ a splitting subset of $\mathcal{S}$. We claim that $\{a\}$ is a formal point of $\mathcal{S}$. Since $\{a\}$ trivially satisfies the first and third condition in Proposition 1.2, it is sufficient to show that $\{a\}$ is splitting. This amounts to showing that $a \triangleleft U \Rightarrow a \in U$ for every $U \subseteq S$. The assumption $a \triangleleft U$ together with $a \triangleleft\{a\}$, which always holds, implies $a \triangleleft U \cap\{a\}$. Together with $a \in Z$, this gives $(U \cap\{a\}) \gamma Z$ because $Z$ is splitting. Hence, in particular, $U \cap\{a\}$ is inhabited, that is, $a \in U$. 
Proposition 2.6 The statement "every sub-locale of a discrete locale is spatial" is equivalent to LEM.

Proof In the presence of LEM, reducibility and spatiality coincide; so one half of the statement follows from Proposition 2.1. As for the other half, we show that the apparently weaker assumption "every closed sub-terminal locale is spatial" already yields LEM. Closed sub-locales of 1 correspond to nuclei of the form $j_{P}(X)=X \cup P$ for $P \in \mathcal{P}(1)$. We claim that the sublocale of fixed points of $j_{P}$ is spatial if and only if $P \cup-P=1$. Of course, the map $j_{P}(Q) \mapsto Q$ is the only potential frame homomorphism from the fixed points of $j_{P}$ to $\mathcal{P}(1)$. This is a well-defined map (and hence a point) if and only if $P=\emptyset$. So spatiality for the sublocale corresponding to $j_{P}$ amounts to the statement $\forall Q, R[(P=\emptyset \Rightarrow Q \subseteq R) \Rightarrow Q \subseteq R \cup P]$, which says that $P \cup-P=1$ (take $Q=1$ and $R=-P$ ).

As a corollary of the previous two propositions, we have that reducibility does not imply spatiality intuitionistically. We will show (paragraph on Baire space at the end of the following section) that spatiality does not imply reducibility either.

\section{The formal cover associated to a tree}

For $S$ a set, let $\operatorname{List}(S)$ be the set of finite lists of elements of $S$, regarded as the full $S$-tree (every node has $S$-many immediate successors). We introduce some notation: \langle\rangle is the empty list, which is the root of the tree, and $\left\langle a_{1}, \ldots, a_{n}\right\rangle$ is the list with elements $a_{1}, \ldots, a_{n} \in S ;|l| \in \mathbb{N}$ is the length of the list $l$; and $l k$ is the concatenation of the two lists $l$ and $k$. We say that $l$ is an initial segment of $l k$, for any $k$, and that $l\langle a\rangle$ is an immediate successor of $l$, for every $a \in S$.

We consider the smallest formal cover $\triangleleft$ on $\operatorname{List}(S)$ such that every node (except the root) is covered by its immediate predecessor as well as by the set of its immediate successors. This is expressed formally by an inductive generation as explained by Sambin [20], Coquand, Sambin, Smith and Valentini [9], and Ciraulo, Maietti and Sambin [4]..$^{5}$ The intuitive idea is that $l \triangleleft U$ holds when every infinite path which

\footnotetext{
${ }^{5}$ It is possible to show that $\triangleleft$ is the smallest relation which satisfies the following conditions: (i) if $l \in U$, then $l \triangleleft U$; (ii) if $l=k\langle a\rangle$ and $k \triangleleft U$, then $l \triangleleft U$; and (iii) if $l\langle a\rangle \triangleleft U$ for all $a \in S$, then $l \triangleleft U$. The existence of such a cover requires a specific mathematical assumption which gives a natural extension of the minimalist foundation.
} 
passes through the node $l$ also passes through some node in $U$. Actually this idea becomes a fact precisely when such a topology is spatial, as we now see.

It can be shown that a subset $T \subseteq \operatorname{List}(S)$ is splitting if and only if

(i) $T$ is closed under initial segments, and

(ii) if $l \in T$, then some immediate successor of $l$ belongs to $T$.

An inhabited $T$ which satisfies (i) is, by definition, a sub-tree of $\operatorname{List}(S) .{ }^{6}$ Clearly, (ii) is a positive way of saying that $T$ has no finite branches, hence no leaves. Therefore, an inhabited splitting subset is what some authors, especially in descriptive set-theory, call a pruned sub-tree of $\operatorname{List}(S)$.

A formal point of $(\operatorname{List}(S), \triangleleft)$ is thus a pruned sub-tree $\alpha \subseteq \operatorname{List}(S)$ which, in addition, satisfies the following: for all $a, b \in S$, if $l\langle a\rangle \in \alpha$ and $l\langle b\rangle \in \alpha$, then $a=b$. Therefore it is (identifiable with) an infinite path.

We say that a sub-tree $T$ of $\operatorname{List}(S)$ is strongly infinite when it contains a pruned sub-tree. Every strongly infinite tree contains arbitrary-long branches, that is, it has infinite height; in particular, it has infinitely many nodes. ${ }^{7}$

Proposition 3.1 For a set $S$, the following are equivalent:

(1) The formal cover on $\operatorname{List}(S)$ is reducible.

(2) Every pruned sub-tree of List $(S)$ contains an infinite path.

(3) Every strongly infinite sub-tree of $\operatorname{List}(S)$ contains an infinite path.

Proof $1 \Rightarrow 2$. If $T$ is pruned, then \langle\rangle$\ltimes_{\triangleleft} T$. By reducibility, there is a formal point $\alpha \subseteq T$, that is, an infinite path in $T$.

$2 \Rightarrow 3$. If $T$ is strongly infinite, then it contains a pruned sub-tree.

$3 \Rightarrow 1$. Assume $l \in T$ with $T$ splitting; so $T$ is pruned. Consider the sub-tree $T_{l}$ given by those lists in $T$ which are comparable with $l$. Note that $T_{l}$ is pruned as well, hence strongly infinite. By assumption, $T_{l}$ contains an infinite path, which necessarily passes through the node $l$. In other words, there is a point $\alpha$ with $l \in \alpha \subseteq T_{l} \subseteq T$.

\footnotetext{
${ }^{6}$ Here we do not require $T$ to be decidable, which, on the contrary, is a quite common requirement in the literature on constructive mathematics.

${ }^{7}$ The converse is true classically (in ZFC set theory), provided that $S$ is finite, that is, every infinite, finitely branching tree is strongly infinite (because of König's Lemma: every infinite, finitely branching tree contains an infinite path). This fact cannot hold constructively: by using a result of Berger, Ishihara and Schuster [3, Proposition 19], one can show that the assumption "every infinite binary tree is strongly infinite" implies LLPO. We thank Tatsuji Kawai for pointing out this result to us.
} 
It is well known that the statement "every pruned tree has an infinite path" is equivalent (over $Z F$ set theory) to Dependent Choice. This fact holds also in the minimalist foundation, as we now show. Dependent Choice (DC) is the assumption that the following principle $\mathrm{DC}(X)$ holds for every set $X$.

$\mathrm{DC}(X)$ : given an element $a \in X$ and a total relation $R \subseteq X \times X$, there is a functional relation $f \subseteq \mathbb{N} \times X$ such that $f(0)=a$ and $(f(n), f(n+1)) \in R$ for all $n \in \mathbb{N}$. $^{8}$

Lemma 3.2 Given a set $S$, consider the following:

(i) $D C(T)$ holds for every pruned sub-tree $T \subseteq \operatorname{List}(S)$.

(ii) The formal cover on $\operatorname{List}(S)$ is reducible.

(iii) $D C(X)$ holds for every $X \subseteq S$.

Then $(i) \Longrightarrow(i i) \Longrightarrow($ iii $)$.

Proof Given a pruned sub-tree $T \subseteq \operatorname{List}(S)$, let us consider the relation $R \subseteq T \times T$ given by all pairs $(l, l\langle a\rangle)$ with $l\langle a\rangle \in T$. Such a relation is total since $T$ is pruned. So $D C(\mathrm{~T})$ provides us with a function $f \subseteq \mathbb{N} \times T$ such that $f(0)=\langle\rangle$ and, for every $n \in \mathbb{N}$, $f(n+1)=f(n)\langle a\rangle$ for some $a \in S$. So $\{f(n) \mid n \in \mathbb{N}\}$ is the required infinite path in $T$.

Given any total relation $R \subseteq X \times X$ and any $a \in X$, we can define inductively a sub-tree $T_{R} \subseteq \operatorname{List}(S)$ by means of the following clauses: (i) \langle\rangle$\in T_{R}$; (ii) $\langle a\rangle \in T_{R}$; and (iii) if $l\langle x\rangle \in T_{R}$ and $(x, y) \in R$, then $l\langle x, y\rangle \in T_{R}$. Clearly $T_{R}$ is pruned precisely because $R$ is total. By Proposition 3.1, there is an infinite path $\alpha \subseteq T_{R}$. The set $\{(|l|, x) \in \mathbb{N} \times X \mid l\langle x\rangle \in \alpha\}$ is a well-defined function which does the job required by $\operatorname{DC}(X)$.

Proposition 3.3 The formal cover on $\operatorname{List}(S)$ is reducible for all sets $S$ if and only if DC holds. ${ }^{9}$

Proof It follows at once from the previous lemma.

\footnotetext{
${ }^{8}$ In the minimalist foundation of Maietti and Sambin [17, 15] the notion of a function from $\mathbb{N}$ to $X$, in the set-theoretic sense of a functional relation $f \subseteq \mathbb{N} \times X$, is kept distinct from that of a sequence of elements of $X$, that is, a term in the type $\mathbb{N} \rightarrow X$. This means that the axiom of unique choice is not included in the "minimalist" system. Thus common formulations of DC, which require the existence of an operation, are definitely stronger than our formulation above.

${ }^{9}$ For partial results in this direction see Sambin [22], Maietti [16], and Rinaldi, Sambin and Schuster [19, Proposition 7.8].
} 
Baire space. Let us have a closer look to the case $S=\mathbb{N}$. Spatiality for such a topology is equivalent to Monotone Bar Induction (see Fourman and Grayson [11] and Gambino and Schuster [14] for details). An inhabited splitting subset $U$ of $(\operatorname{List}(\mathbb{N}), \triangleleft)$ is a spread. ${ }^{10}$ So, the Baire formal cover is reducible precisely when every spread contains an infinite path. (See Maietti and Sambin [18] for more on this topic; in the decidable case, this is sometimes called Brouwer's Spread Lemma.)

As a corollary we get that spatiality does not imply reducibility. We thank Tatsuji Kawai for suggesting the following argument. In [12, Theorem 3.4] Fourman and Hyland show that the internal theory of the topos $S h(\mathbb{R})$ of sheaves over the reals satisfies the principle of Monotone Bar Induction, which expresses spatiality of the Baire space. On the contrary, the axiom of choice from numbers to numbers AC-NN fails in the internal logic of $S h(\mathbb{R})$ because the Dedekind and Cauchy reals do not coincide there [12, page 289]. Recall that AC-NN is the following: given a total relation $R \subseteq \mathbb{N} \times \mathbb{N}$, there exists a functional relation $f \subseteq \mathbb{N} \times \mathbb{N}$ such that $(n, f(n)) \in R$ for all $n \in \mathbb{N}$. We want to show that AC-NN becomes provable if the Baire space is reducible. Indeed, given a total relation $R \subseteq \mathbb{N} \times \mathbb{N}$, one can define a spread $U$ by induction by means of the following clauses: (i) \langle\rangle$\in U$; and (ii) if $l \in U$ and $(|l|, x) \in R$, then $l\langle x\rangle \in U$. If $\alpha$ is a path (= ideal point) in $\mathrm{U}$, then $\{(|l|, x) \in \mathbb{N} \times \mathbb{N} \mid l\langle x\rangle \in \alpha\}$ is a well-defined function $f$ with $(n, f(n)) \in R$ for all $n \in \mathbb{N}$.

Cantor space. By specializing to the case $S=2$ we get Cantor formal cover $(\operatorname{List}(2), \triangleleft)$. By a result of Fourman and Grayson [11], such a locale is spatial precisely if the full Fan Theorem holds (for arbitrary, not necessarily decidable, bars). See Gambino and Schuster [14] for details.

We now can add a dual statement. Reducibility of Cantor space amounts to the following: every pruned binary tree contains an infinite path. By Proposition 3.1, this is equivalent to the statement every strongly infinite binary tree contains an infinite path, which is manifestly a weak version of the Weak König's Lemma.

\section{Positive Topologies}

In a concrete space $\mathcal{X}=(X, \Vdash, S)$, also the closed subsets of $X$ have their "formal companions" in $S$. In order to see this, we first define

$$
\text { rest } U=\{x \in X \mid \diamond x \subseteq U\}
$$

\footnotetext{
${ }^{10}$ The usual definition of a spread includes decidability of $U$; see, for instance, Troelstra and van Dalen [25].
} 
where $\diamond x=\{a \in S \mid x \Vdash a\}$ is the system of (indexes of) basic neighbourhoods of $x$. When $U$ ranges over the subsets of $S$, the subsets rest $U$ of $X$ describe all closed sets. Each closed set, however, can be the image of several subsets, unless a canonical choice is made as follows. Let us consider the following positivity relation between elements and subsets of $S$. For $a \in S$ and $U \subseteq S$, we put

$$
a \ltimes \mathcal{X} U \Leftrightarrow \operatorname{ext} a \gamma \operatorname{rest} U \Leftrightarrow(\exists x \in X)(a \in \diamond x \subseteq U) .
$$

As shown by the second author [22], rest becomes one-to-one when restricted to subsets of the form $\left\{a \in S \mid a \ltimes_{\mathcal{X}} U\right\}$. These we call formal closed subsets.

The following definition from [22] captures much of the structure $(S, \triangleleft \mathcal{X}, \ltimes \mathcal{X})$ induced by a concrete space.

Definition 4.1 A positive topology is a triple $\mathcal{S}=(S, \triangleleft, \ltimes)$ where $\triangleleft$ is a formal cover and $\ltimes \subseteq S \times \mathcal{P}(S)$ satisfies the following conditions for all $a \in S$ and $U, V \subseteq S$ :

(1) if $a \ltimes U$, then $a \in U$;

(2) if $a \ltimes U$ and $b \in V$ for all $b$ with $b \ltimes U$, then $a \ltimes V$; and

(3) if $a \triangleleft U$ and $a \ltimes V$, then $u \ltimes V$ for some $u \in U$ (compatibility).

The relation $\ltimes$ is called a positivity relation and subsets of the form $\{a \in S \mid a \ltimes U\}$ are called formal closed. For $\mathcal{X}$ a concrete space, $\mathcal{S}_{\mathcal{X}}=(S, \triangleleft \mathcal{X}, \ltimes \mathcal{X})$ is a positive topology. Positive topologies of this kind are called representable (by a concrete space).

Positive topologies from an algebraic point of view. Now, let $(S, \triangleleft, \ltimes)$ be a positive topology and let $L$ be the complete lattice (of formal open subsets) presented by $(S, \triangleleft)$. What does $\ltimes$ and the formal closed subsets present? ${ }^{11}$ The compatibility rule says precisely that every formal closed subset $F$ is splitting (Definition 2.3).

Splitting subsets are closed under unions and so they form a suplattice as well, called $\operatorname{Split}(S, \triangleleft)$. The collection of formal closed subsets with respect to the given $\ltimes$ turns out to be a sub-suplattice of $\operatorname{Split}(S, \triangleleft)$. Conversely, let $M$ be a sub-suplattice of $\operatorname{Split}(S, \triangleleft)$. Then the relation $a \ltimes U \Leftrightarrow(\exists Z \in M)(a \in Z \subseteq U)$ is a positivity relation compatible with $\triangleleft$ and, moreover, the corresponding collection of formal closed subsets is precisely $M .{ }^{12}$

\footnotetext{
${ }^{11}$ See Ciraulo and Vickers [8] for a more detailed answer to this question, especially in the case of locales.

${ }^{12}$ This argument is impredicative unless $M$ is set-based. In general, however, there is no reason to assume that the suplattice of formal closed subsets is set-based. Therefore, we could say that a positivity relation is a "more concrete" object than the suplattice of splitting subsets it presents.
} 
Classically, $\operatorname{Split}(S, \triangleleft)$ is isomorphic to $L^{o p}$ ( $L$ with reversed order). Hence a positivity relation on a suplattice $L$ is, classically, just a sub-suplattice of $L^{o p}$, that is, the opposite of a suplattice quotient of $L$.

On morphisms. In this paper we need not use the notion of a morphism between positive (basic) topologies [22]. So, we skip a precise definition and we content ourselves with the following idea. An arrow between positive (basic) topologies, from $\mathcal{S}_{1}$ to $\mathcal{S}_{2}$ say, corresponds to a frame (suplattice) homomorphism $f$ from the formal open subsets of $\mathcal{S}_{2}$ to the formal open subsets of $\mathcal{S}_{1}$ (note the opposite direction). In addition, $f$ has to "respect" the positivity relations, in the following sense. If we regard splitting subsets, and hence formal closed subsets, as join-preserving maps to $\mathcal{P}(1)$ (as explained above), then $U \circ f$ must be one of the formal closed subsets of $\mathcal{S}_{2}$ whenever $U$ is formal closed in $\mathcal{S}_{1}$ (which makes sense since $U \circ f$ is splitting anyway).

Locales as "thick" positive topologies. In a positive topology $(S, \triangleleft, \ltimes)$ the following is always true: if $a \ltimes U$, then $a \in V \subseteq U$ for some splitting subset $V$ (take $V=$ $\{x \in S \mid x \ltimes U\}$ ). Let us consider the relation

$$
a \ltimes_{\triangleleft} U \quad \stackrel{\text { def }}{\Longleftrightarrow} \quad(\exists V \text { splitting })(a \in V \subseteq U)
$$

(which makes sense in an impredicative setting). In [7], we showed that $\ltimes_{\triangleleft}$ is a positivity relation, actually the greatest among the positivity relations which are compatible with $\triangleleft$.

Definition 4.2 A positive topology $(S, \triangleleft, \ltimes)$ is called thick (or "localic") if $\ltimes=\ltimes_{\triangleleft}$, which happens precisely when $a \ltimes U$ follows from the assumption that $a \in V \subseteq U$ for some splitting subset $V$.

So a thick topology is one in which formal closed subsets and splitting subsets coincide. Thick positive topologies, that is, those of the form $\left(S, \triangleleft, \ltimes_{\triangleleft}\right)$, are impredicatively the same thing as locales. In fact the notion of a morphism between thick positive topologies reduces to that between the corresponding locales of formal opens. Also in a predicative setting the examples of thick positive topologies abound. For instance Alexandrov topologies are always thick (see 4.3 below). ${ }^{13}$ Classically (and predicatively) every representable positive topology is thick.

\footnotetext{
${ }^{13}$ In general, if the cover relation $\triangleleft$ is defined by induction (like in the case of trees; see Section 3 above), then it is possible to define a positivity relation $\ltimes=\ltimes_{\triangleleft}$ by coinduction.
} 


\subsection{Spatiality and reducibility for Positive topologies}

By taking inspiration from the properties of a subset of the form $\diamond x$ for $x \in X$ in a concrete space $\mathcal{X}$, the second author reached the following definition of an ideal point [22].

Definition 4.3 Let $\mathcal{S}=(S, \triangleleft, \ltimes)$ be a basic or positive topology. A subset $\alpha \subseteq S$ is an ideal point of $\mathcal{S}$ if

(i) $\alpha$ is inhabited,

(ii) $\alpha$ is formal closed, and

(iii) whenever $\{a, b\} \subseteq \alpha$, there is $c \in \alpha$ with $c \triangleleft\{a\}$ and $c \triangleleft\{b\}$.

We write IPt $\mathcal{S}$ for the collection of ideal points of $\mathcal{S}$.

Ideal points correspond to global elements in the category of positive topologies. Note that (ii) is stronger than the usual requirement for a formal point of $(S, \triangleleft)$, namely that $\alpha$ is a splitting subset; so, the ideal points of $(S, \triangleleft, \ltimes)$ are generally fewer than the formal points of $(S, \triangleleft)$. However, the ideal points of a thick positive topology $\left(S, \triangleleft, \ltimes_{\triangleleft}\right)$ are precisely the formal points of $(S, \triangleleft)$.

Note that $\operatorname{IPt}(\mathcal{S})$ need not be a set predicatively. Impredicatively, on the contrary, one always has a concrete space $(\operatorname{IPt}(\mathcal{S}), \ni, S)$ and hence the induced positive topology $\left(S, \triangleleft_{\mathrm{IPt}}, \ltimes_{\mathrm{IPt}}\right)$. Note that the two inclusions $\triangleleft \subseteq \triangleleft_{\mathrm{IPt}}$ and $\ltimes_{\mathrm{IPt}} \subseteq \ltimes$ always hold since every ideal point is formal closed, hence splitting.

The notion of a reducible topology was originally introduced by the second author [22] in the framework of positive topologies, where it naturally arises.

Definition 4.4 A positive topology $(S, \triangleleft, \ltimes)$ is called

spatial if $\triangleleft_{\mathrm{IPt}}=\triangleleft$, which happens precisely when $a \triangleleft U$ follows from the assumption that $a \in \alpha \Rightarrow \alpha \gamma U$ for every ideal point $\alpha$;

reducible if $\ltimes_{\mathrm{IPt}}=\ltimes$, which happens precisely when $a \ltimes U$ implies the existence of an ideal point $\alpha$ such that $a \in \alpha \& \alpha \subseteq U$;

bi-spatial if it is both spatial and reducible.

This terminology is coherent with that of the previous sections. Indeed, spatiality and reducibility for a thick topology $\left(S, \triangleleft, \ltimes_{\triangleleft}\right)$ are clearly equivalent to those for $(S, \triangleleft)$. Note that if $(S, \triangleleft)$ is spatial, then $(S, \triangleleft, \ltimes)$ is spatial for every $\ltimes$, because of the chain of inclusions $\triangleleft \subseteq \triangleleft_{\mathrm{IPt}} \subseteq \triangleleft_{\mathrm{Pt}}$. 
A positive topology is reducible precisely when every formal closed subset is a union of ideal points (compare this to the remark after Definition 2.4), that is, when the notion of a formal closed set is "reducible" to that of a point.

On the relation between reducibility and spatiality for positive topologies. In Subsection 2.1 above, we proved that reducibility and spatiality for a locale are not comparable intuitionistically, although they coincide classically.

For positive topologies, in general, the implication "reducibility implies spatiality" also fails classically. Let $(S, \triangleleft)$ be a non spatial formal cover and consider the positive topology $\left(S, \triangleleft, \ltimes_{\mathrm{Pt}}\right)$. One can show that its ideal points are precisely the formal points of $(S, \triangleleft)$ and hence $\left(S, \triangleleft, \ltimes_{\mathrm{Pt}}\right)$ is reducible; but not spatial. Note that $\left(S, \triangleleft, \ltimes_{\mathrm{Pt}}\right)$ is different from the thick positive topology $\left(S, \triangleleft, \ltimes_{\triangleleft}\right)$.

On the other hand, spatial positive topologies are always reducible in a classical framework. Let $\mathcal{S}=(S, \triangleleft, \ltimes)$ be spatial and assume $a \ltimes U$. If there were no ideal point $\alpha$ such that $a \in \alpha \subseteq U$, then $a \in \alpha$ would imply $\alpha \gamma-U$ for all ideal points $\alpha$, and hence $a \triangleleft-U$ would hold because $\mathcal{S}$ is spatial. By compatibility, there should be some $b \in-U$ such that $b \ltimes U$, hence $b \in U$. This is a contradiction.

Positive topologies and inclusion between spaces. Every inclusion of spaces corresponds to a particular positive topology. To see this, let $Y$ be a subset of $X$, which gives a subspace $\mathcal{Y}$ of $\mathcal{X}$ in the obvious way. Now $(S, \triangleleft \mathcal{X}, \ltimes \mathcal{Y})$ is a completely legitimate positive topology in which the formal closed subsets correspond to the closed subsets of $Y$. In general, a binary positivity predicate is a way to select a collection of points (see Sambin and Trentinaglia [23], Ciraulo and Vickers [8], and Section 4.1 below).

Each positive topology $\mathcal{S}=(S, \triangleleft, \ltimes)$ determines a collection of ideal points $\operatorname{IPt}(\mathcal{S})$, which is a sub-collection of all formal points $\operatorname{Pt}\left(\mathcal{S}^{\prime}\right)$ of the locale $\mathcal{S}^{\prime}=(S, \triangleleft)$. This gives impredicatively an inclusion of spaces $\operatorname{IPt}(\mathcal{S})=Y \subseteq X=\operatorname{Pt}\left(\mathcal{S}^{\prime}\right)$. In turn, this inclusion gives back a positive topology $\left(S, \triangleleft_{X}, \ltimes_{Y}\right)$ whose formal open subsets correspond to the opens of $X$, and the formal closed subsets correspond to the closed subsets of $Y$. Note that such a positive topology coincides with $\mathcal{S}$ precisely when $\mathcal{S}$ is reducible and $\mathcal{S}^{\prime}$ is spatial. This happens in particular when $\mathcal{S}$ is bi-spatial, since the chain of inclusions $\triangleleft \subseteq \triangleleft_{X} \subseteq \triangleleft_{Y}$ always holds.

\subsection{Bi-spatiality and the "size" of the collection of points}

Given a concrete space $\mathcal{X}=(X, \Vdash, S)$ and a (concrete) point $x \in X$, the subset $\diamond x$ $=\{a \in S \mid x \Vdash a\}$ is an ideal point of $\mathcal{S}_{\mathcal{X}}=(S, \triangleleft \mathcal{X}, \ltimes \mathcal{X})$, hence a formal point of 
$(S, \triangleleft \mathcal{X})$. When $\diamond$ is a bijection between $X$ and $\operatorname{IPt}\left(\mathcal{S}_{\mathcal{X}}\right)$, then $\mathcal{X}$ is called weakly sober. It is called sober if $\diamond$ is a bijection between $X$ and $\operatorname{Pt}(S, \triangleleft \mathcal{X})$, in which case $\operatorname{IPt}\left(\mathcal{S}_{\mathcal{X}}\right)=\operatorname{Pt}(S, \triangleleft \mathcal{X})$ and so $\mathcal{X}$ is also weakly sober.

More explicitly, the difference between ideal points of $\mathcal{S}_{\mathcal{X}}$ and formal points of $(S, \triangleleft \mathcal{X})$ is the following. The latter correspond to completely-prime filters of opens: given $\alpha$ define $F_{\alpha}=\{\operatorname{ext} U \mid U \gamma \alpha\}$; and given $F$ define $\alpha_{F}=\{a \in S \mid \operatorname{ext} a \in F\}$. An ideal point of $\mathcal{S}_{\mathcal{X}}$ corresponds instead to a closed subset $C \subseteq X$ which is irreducible in the following sense $\mathrm{e}^{14}$

$$
\operatorname{ext} U \varnothing C \& \operatorname{ext} V \gamma C \Rightarrow(\operatorname{ext} U \cap \operatorname{ext} V) \varnothing C \text { for all } U, V
$$

(classically this is equivalent to the usual notion of an irreducible closed subset). The correspondence is as follows. To each $\alpha$ we associate rest $\alpha$ and, vice versa, to each $C$ we associate $\diamond C=\{a \in S \mid \operatorname{ext} a \gamma C\}$. Classically, of course, the two notions of a point are equivalent and so sobriety and weak sobriety coincide. Intuitionistically, the picture is the following (see Aczel and Fox [2] and Fox [13]): every $T_{2}$ space is weakly sober, while the implication "every $T_{2}$ space is sober" entails LPO.

Impredicatively, a positive topology $\mathcal{S}$ is bi-spatial if and only if it is representable by a concrete space, namely $(\operatorname{IPt}(\mathcal{S}), \ni, S)$. A predicative version follows (cf Proposition 6.1).

Proposition 4.5 For every positive topology $\mathcal{S}$, the following are equivalent:

(1) $\mathcal{S}$ is representable by a concrete space $\mathcal{X}$.

(2) $\mathcal{S}$ is bi-spatial and $\operatorname{IPt}(\mathcal{S})$ is set-based. ${ }^{15}$

Moreover the following are also equivalent:

$1^{\prime} . \mathcal{S}$ is representable by a weakly sober space $\mathcal{X}$.

$2^{\prime} . \mathcal{S}$ is bi-spatial and $\operatorname{IPt}(\mathcal{S})$ is a set. ${ }^{16}$

Proof Assume $\mathcal{S}=\mathcal{S}_{\mathcal{X}}$, with $\mathcal{X}$ a concrete space. If $a \triangleleft_{\mathrm{IPt}} U$, then in particular $a \in \diamond x \Rightarrow U \curlyvee \diamond x$ for all $x \in X$. This is nothing but $a \triangleleft \mathcal{X} U$, which coincides with

\footnotetext{
${ }^{14}$ Such a constructive notion of an irreducible subset is taken from [22], where it appears under the name "convergent subset".

${ }^{15}$ In this case, with "set-based" we mean that there exists a set of ideal points which generate all the others by union.

${ }^{16}$ See Curi [10] and Aczel and Curi [1] (and the literature cited therein) for more information on when $\operatorname{IPt}(\mathcal{S})$ is a set. Clearly, if $\operatorname{IPt}(\mathcal{S})$ is a set, then it is also set-based; and the converse holds provided that the topology on $\operatorname{IPt}(\mathcal{S})$ is $T_{1}$.
} 
$a \triangleleft U$ by assumption. Similarly, $a \ltimes U$ means $a \in \diamond x \subseteq U$ for some $x \in X$, because $\ltimes=\ltimes \mathcal{X}$. So $\mathcal{S}$ is reducible because every $\diamond x$ is an ideal point. Finally, every $\alpha$ is the union of the $\diamond x$ 's contained in it and so $\{\diamond x \mid x \in X\}$ is a base for $\operatorname{IPt}(\mathcal{S})$ (indexed by the set $X)$.

Conversely, if $\operatorname{IPt}(\mathcal{S})$ is set-based with base $\left\{\alpha_{x} \mid x \in X\right\}$, then we consider the triple $\mathcal{X} \equiv(X, \Vdash, S)$, where $x \Vdash a$ is $a \in \alpha_{x}$. We claim that $\mathcal{S}=\mathcal{S}_{\mathcal{X}}$ and that $\mathcal{X}$ is a concrete space. Clearly $a \triangleleft U$ implies $a \triangleleft \mathcal{X} U$ because every $\alpha_{x}$ is splitting. The opposite direction holds because $\mathcal{S}$ is spatial: if $a \in \alpha_{x}$ implies $U \gamma \alpha_{x}$ for all $x \in X$, then the same holds for any ideal point $\alpha$, since the $\alpha_{x}$ 's are a base for $\operatorname{IPt}(\mathcal{S})$; so $a \triangleleft \mathcal{X} U$ implies $a \triangleleft_{\mathrm{IPt}} U$. The proof of $\ltimes=\ltimes_{\mathcal{X}}$ is similar. Indeed $\ltimes \mathcal{X} \subseteq \ltimes$ holds because every $\alpha_{x}$ is formal closed. Since $\mathcal{S}$ is reducible, $\ltimes \subseteq \ltimes \mathcal{X}$ is equivalent to $\ltimes_{\mathrm{IPt}} \subseteq \ltimes_{\mathcal{X}}$, which holds because the $\alpha_{x}$ 's form a base for the ideal points. Finally, $\mathcal{X}$ turns out to be a concrete space since every $\diamond x=\alpha_{x}$ is an ideal point of $\mathcal{S}_{\mathcal{X}}$.

We now come to the second part of the statement. Recall that $\mathcal{X}$ is weakly sober precisely when the mapping $x \mapsto \diamond x$ is a bijection between $X$ and $\operatorname{IPt}\left(\mathcal{S}_{\mathcal{X}}\right)$. So if $\mathcal{X}$ is weakly sober, then $\operatorname{IPt}\left(\mathcal{S}_{\mathcal{X}}\right)$ is a set. Conversely, if $\operatorname{IPt}(\mathcal{S})$ is a set, then the structure $(\operatorname{IPt}(\mathcal{S}), \ni, S)$ is a completely legitimate concrete space representing $\mathcal{S}$, as it is easy to check.

Note that the following become equivalent within an impredicative framework: (i) $\mathcal{S}$ is bi-spatial; (ii) $\mathcal{S}=\mathcal{S}_{\mathcal{X}}$ for some concrete space $\mathcal{X}$; (iii) $\mathcal{S}=\mathcal{S}_{\mathcal{X}}$ for some weakly sober concrete space $\mathcal{X}$. This does not hold predicatively, as shown by the following example.

The Sierpinski formal cover. Let us consider the formal cover $\mathbb{S}$ induced by the Sierpinski space $(2, \leq, 2)$, where $2=\{0,1\}$ and $0 \leq 1$. Here $\alpha \subseteq 2$ is a formal point if and only if $1 \in \alpha$. So we can define two maps between $\operatorname{Pt}(\mathbb{S})$ and $\mathcal{P}(1)$, where $1=\{0\}$, by putting $U_{\alpha}=\{0\} \cap \alpha$ and $\alpha_{U}=U \cup\{1\}$. Clearly these are inverse to each other and so $\operatorname{Pt}(\mathbb{S})$ can be identified with $\mathcal{P}(1)$. Predicatively, $\mathcal{P}(1)$ is not a set. In view of Proposition 4.5, this shows that $\mathbb{S}$ cannot be represented by a (weakly) sober space. So a positive topology induced by a concrete space need not be representable by a (weakly) sober space, contrary to what happens impredicatively.

\subsection{An example: Alexandrov topologies}

If $\leq$ be a preorder (reflexive and transitive relation) on a set $S$, then $(S, \leq, S)$ is a concrete space, that is, the subsets of the form $\downarrow a=\{x \in S \mid x \leq a\}$, for $a \in S$, form a 
base for a topology on $S$. Therefore we can consider the induced bi-spatial positive topology, say $\left(S, \triangleleft_{\leq}, \ltimes_{\leq}\right)$. For $a \in S$ and $U \subseteq S$ we then have

$$
\begin{aligned}
& a \triangleleft_{\leq} \quad \Longleftrightarrow \downarrow a \subseteq \bigcup_{u \in U} \downarrow u \quad \Longleftrightarrow \uparrow a \gamma U \\
& a \ltimes \leq U \Longleftrightarrow \uparrow x(a \in \uparrow x \subseteq U) \Longleftrightarrow \uparrow a \subseteq U
\end{aligned}
$$

where $\uparrow x=\{a \in S \mid x \leq a\}$. The formal open (as well as the concrete open) subsets are the lower sets with respect to $\leq$. Dually, the formal closed (as well as the concrete closed) subsets are precisely the up upper sets. The ideal points turn out to be the inhabited, upper sets which are also downward-directed. These not necessarily are of the form $\uparrow a$ with $a \in S$; for example, if $(S, \leq)$ is a linear, bottom-less order, then $S$ itself is a formal point.

As a topological space, $(S, \leq, S)$ is just the set $S$ equipped with the so-called "lower topology" with respect to $\leq$. This is nothing but the Alexandrov topology on $(S, \geq)$, where $\geq$ is the specialization preorder, that is, $x \geq y$ if and only if $x$ belongs to the closure of $y$. In other words, positive topologies of the form $\left(S, \triangleleft_{\leq}, \ltimes_{\leq}\right)$correspond to Alexandrov spaces, that is, spaces in which any intersection of open subsets is open. (See Sambin, Valentini and Virgili [24] for the connection between this kind of topologies and Scott domains.)

More generally, if $\leq_{1}$ and $\leq_{2}$ are two partial orders on $S$, then one could wonder whether $\left(S, \triangleleft_{\leq_{1}}, \ltimes_{\leq_{2}}\right.$ ) is a positive topology, that is, whether the compatibility condition holds between $\triangleleft_{\leq_{1}}$ and $\ltimes_{\leq_{2}}$. This happens precisely when $\leq_{1} \subseteq \leq_{2}$. For, whenever $a \leq_{1} b$, compatibility applied to the premises $a \triangleleft_{\leq_{1}}\{b\}$ and $a \ltimes_{\leq_{2}} \uparrow_{2} a$ gives $b \ltimes_{\leq_{2}} \uparrow_{2} a$; this is $\uparrow_{2} b \subseteq \uparrow_{2} a$, that is, $a \leq_{2} b$. Conversely, if $\leq_{1} \subseteq \leq_{2}$, then $\triangleleft_{\leq_{1}} \subseteq \triangleleft_{\leq_{2}}$ as well; so compatibility between $\triangleleft_{\leq_{1}}$ and $\ltimes_{\leq_{2}}$ follows from that between $\triangleleft_{\leq_{2}}$ and $\ltimes_{\leq_{2}}$. Positive topologies of this form are called elementary.

In the case of an elementary topology, the formal open subsets are the lower subsets with respect to $\leq_{1}$ and the formal closed subsets are the upper subsets with respect to $\leq_{2}$. Note that the splitting subsets are upper subsets as well, but with respect to $\leq_{1}$. Finally, ideal points turn out to be the inhabited, upper subsets with respect to $\leq_{2}$ which are also downward-directed with respect to $\leq_{1}$.

Proposition 4.6 For an elementary positive topology $\mathcal{S}=\left(S, \triangleleft_{\leq_{1}}, \ltimes_{\leq_{2}}\right)$ the following are equivalent:

(1) $\leq_{1}=\leq_{2}$.

(2) $\mathcal{S}$ is representable by a concrete space.

(3) $\mathcal{S}$ is spatial. 
(4) $\mathcal{S}$ is thick.

Furthermore, each of the above items implies that $\mathcal{S}$ is reducible, while the converse holds provided that $\leq_{2}$ (and hence $\leq_{1}$ ) is antisymmetric.

Proof $1 \Rightarrow 2$ because, by its very definition, a positive topology of the form $\left(S, \triangleleft_{\leq}, \ltimes_{\leq}\right)$is represented by the concrete space $\mathcal{X}=(S, \leq, S)$.

$2 \Rightarrow 3$ by Proposition 4.5. Actually 2 implies that $\mathcal{S}$ is bi-spatial, hence reducible, which is a piece of the second part of the statement.

$3 \Rightarrow 4$. In order to show that splitting subsets and formal closed subsets coincide, it is sufficient to show that $\leq_{1}=\leq_{2}$ (so we are actually proving that $3 \Rightarrow 1 \Rightarrow 4$ ). If $a \leq_{2} b$, then the implication $a \in \uparrow_{2} U \Rightarrow b \in \uparrow_{2} U$ holds for every $U$. Since the $\uparrow_{2} U$ 's are the formal closed subsets, this gives $a \triangleleft_{\leq_{1}}\{b\}$ by 2 , which means $a \leq_{1} b$.

$4 \Rightarrow 1$. For any given $a \in S$, we have $a \in \uparrow_{1} a \subseteq \uparrow_{2} a$. Since $\uparrow_{1} a$ is splitting and $\mathcal{S}$ is thick, we get $a \ltimes_{\leq_{2}} \uparrow_{1} a$, that is, $\uparrow_{2} a \subseteq \uparrow_{1} a$. This means that $a \leq_{2} b$ implies $a \leq_{1} b$ for all $a, b \in S$.

We now come to the last part of the statement. For every $a, \uparrow_{2} a \subseteq \uparrow_{2} a$ means that

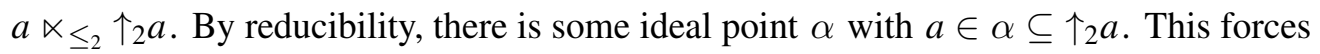
$\alpha$ to be just $\uparrow_{2} a$ because $\alpha$ is upward closed w.r.t. $\leq_{2}$. Therefore reducibility implies that every $\uparrow_{2} a$ is an ideal point. Now assume $a \leq_{2} b$. As $\{a, b\} \subseteq \uparrow_{2} a$, there is $c$ such that (i) $c \triangleleft_{\leq_{1}}\{a\}$, (ii) $c \triangleleft_{\leq_{1}}\{b\}$ and (iii) $c \in \uparrow_{2} a$. Item (i) means $c \leq_{1} a$, from which $c \leq_{2} a$ follows. This together with (iii) gives $c=a$ by antisymmetry of $\leq_{2}$. Hence $a \leq{ }_{1} b$ by (ii).

The preorder $\leq_{2}$ being antisymmetric is a necessary condition to prove the equivalence between reducibility and the other items, as it is shown by the following example. Let $\left(S, \leq_{1}\right)$ be a downward-directed poset with at least two distinct elements. By taking $\leq_{2}$ to be the always true binary relation on $S$, one gets an elementary positive topology which is reducible, because $S$ is the only inhabited formal closed subset and the only ideal point, but not spatial. This shows again that "reducibility implies spatiality" fails for positive topologies, in general (also classically).

\section{On a strong form of overtness}

In the intuitionistic theory of locales, the notion of overt (or open) locale is of some importance. A locale $L$ is overt if the unique frame homomorphism $X \rightarrow \mathcal{P}(1)$ has 
a left adjoint $\operatorname{Pos}_{L}$ as a monotone map. Classically, every locale is overt. Even intuitionistically $\operatorname{Pos}_{L}(x) \neq 1$ is equivalent to $\operatorname{Pos}_{L}(x)=0$, which in turn is equivalent to $x=0$. However $\operatorname{Pos}_{L}(x)=1$ is intuitionistically stronger than $x \neq 0$ and so $\operatorname{Pos}_{X}(x)=1$ can be read as a positive way to express that $x$ is different from 0 . Its predicative counterpart is given by the notion of a formal topology (with a unary positivity predicate), as defined by the second author [20]. This is defined as a triple $(S, \triangleleft, P o s)$ where $\triangleleft$ is a formal cover and $\operatorname{Pos}$ is a predicate on $S$ such that (i) $\{a \in S \mid \operatorname{Pos}(a)\}$ is a splitting subset and (ii) $(\operatorname{Pos}(a) \Rightarrow a \triangleleft U) \Rightarrow a \triangleleft U$ for any given $a \in S$ and $U \subseteq S$.

Definition 5.1 We say that a locale $L$ is strongly overt when $x \leq y$ follows from the assumption that $\varphi(x)=1 \Rightarrow \varphi(y)=1$ for every join-preserving map $\varphi: L \rightarrow \mathcal{P}(1)$. Accordingly, we say that a formal cover $(S, \triangleleft)$ is strongly overt if $a \triangleleft U$ follows from the assumption that $a \in Z \Rightarrow U \gamma Z$ for every splitting subset $Z$.

The term is justified by the following proposition, which we prove in the wider framework of positive topologies (recall that locales can be identified with thick positive topologies, and every splitting subset is formal closed in that case).

Definition 5.2 We say that a positive topology $(S, \triangleleft, \ltimes)$ is strongly overt if $a \triangleleft U$ follows from the assumption that $a \in V \Rightarrow U \curlyvee V$ for every formal closed subset $V .{ }^{17}$

Proposition 5.3 Let $\mathcal{S}$ be a positive topology.

(1) If $\mathcal{S}$ is strongly overt, then it is overt.

(2) If $\mathcal{S}$ is spatial, then it is strongly overt.

(3) If $\mathcal{S}$ is strongly overt and reducible, than it is spatial.

Proof (1) Define $\operatorname{Pos}(a)$ as $a \ltimes S$ and assume $a \ltimes S \Rightarrow a \triangleleft U$. We claim that $a \in V \Rightarrow U \varnothing V$ for every formal closed subset $V$, which will give $a \triangleleft U$ by density. From $a \in V$ we have $a \ltimes S$ because $V$ is formal closed. So $a \triangleleft U$ by assumption. By using $a \in V$ again, we get $U \varnothing V$ by compatibility.

(2) Recall that $\mathcal{S}$ is spatial when $a \triangleleft U$ follows from the assumption that $a \in \alpha \Rightarrow U \gamma \alpha$ for every ideal point $\alpha$. And every ideal point is formal closed by definition.

(3) Assume $a \in \alpha \Rightarrow U \varnothing \alpha$ for every ideal point $\alpha$. We have to show that $a \triangleleft U$. By strong overtness, it is sufficient to show that $a \in V \Rightarrow U \gamma V$ for every formal closed subset $V$. So let $a \in V$. By reducibility, there exists an ideal point $\alpha$ with $a \in \alpha \subseteq V$. So $U \gamma \alpha$ by assumption and hence $U \gamma V$ because $\alpha \subseteq V$.

\footnotetext{
${ }^{17}$ Such positive topologies are called "reduced" in Ciraulo and Sambin [7], and "dense" in Sambin [22].
} 
In particular, the following equivalence holds for every positive topology:

$$
\text { bi-spatial } \Longleftrightarrow \text { strongly overt \& reducible }
$$

Hence we obtain (see Proposition 4.5) the following "minimal" characterization of those positive topologies which come from a concrete space:

$$
\mathcal{S} \text { representable by a concrete space } \Longleftrightarrow\left\{\begin{array}{l}
\operatorname{IPt}(\mathcal{S}) \text { set-based } \\
\mathcal{S} \text { strongly overt } \\
\mathcal{S} \text { reducible }
\end{array}\right.
$$

Classically, every locale is strongly overt, because $x \mapsto(x \not \leq b)$ preserves joins. More generally, we obtained the following result in [7].

Proposition 5.4 In the presence of LEM, a positive topology is strongly overt if and only if it is thick.

Proof A thick positive topology $\mathcal{S}$ can be identified with a formal cover $(S, \triangleleft)$, and the formal closed subsets of $\mathcal{S}$ are precisely the splitting subsets of $(S, \triangleleft)$. Assume $a \in V \Rightarrow U \curlyvee V$ for every splitting subset $V$. We have to show that $a \triangleleft U$. Classically, $\{x \in S \mid \neg(x \triangleleft U)\}$ is splitting. So if $a \triangleleft U$ were false, then $\neg(u \triangleleft U)$ would hold for some $u \in U$. This is a contradiction.

Conversely, let $Z$ be splitting in $(S, \triangleleft)$. We have to show that $Z$ is formal closed in $\mathcal{S}=(S, \triangleleft, \ltimes)$ provided that $\mathcal{S}$ is strongly overt. By way of contradiction, assume $Z$ is not formal closed, so that there is some $a \in Z$ such that $\neg(a \ltimes Z)$. We first claim that $a \triangleleft-Z$, where $-Z$ is the set-theoretic complement of $Z$. By strong density, it is sufficient to show that $a \in V \Rightarrow-Z \gamma V$, that is $a \in V \Rightarrow \neg(V \subseteq Z)$, for every formal closed subset $V$. So let $a \in V$ with $V$ formal closed. If $V \subseteq Z$ were true, then we would have $a \ltimes V$, and $b \in Z$ for all $b$ with $b \ltimes V$; therefore we would get $a \ltimes Z$ by the definition of a positivity relation (Definition 4.1), which contradicts the assumption. This proves that $a \triangleleft-Z$. Since $Z$ is splitting, we get $Z \gamma-Z$. This is a contradiction.

Summing up, in the presence of LEM we have got the following picture for a positive topology $\mathcal{S}$ :

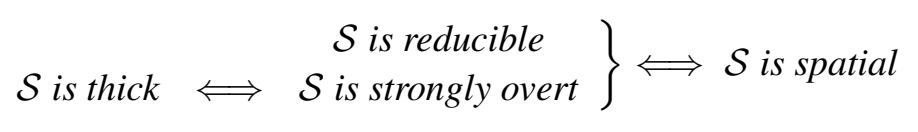

Intuitionistically, both the statement "every strongly overt positive topology is thick" and its converse "every locale is strongly overt" are equivalent to LEM. The interested 
reader can turn the following sketch into a detailed proof, which can be found in [7]. For $p$ any given proposition, consider the triple $\left(\{0,1\}, \in, \ltimes^{p}\right)$ where $0 \ltimes^{p} U$ is $0 \in U$, and $1 \ltimes^{p} U$ is the conjunction of $1 \in U$ and $p \vee(p \rightarrow(0 \in U))$; then show that this is a strongly overt positive topology, which is thick precisely when $p \vee \neg p$ holds. As for the other statement, consider the closed sub-terminal locale $\left(\{0\}, \triangleleft_{p}\right)$, where $0 \triangleleft_{p} U$ if and only if $(0 \in U) \vee p$; show that it is strongly overt precisely when $p \vee \neg p$ holds.

The intuitionistic failure of the previous proposition can be understood from a different perspective. If every strongly overt positive topology were thick, then every bi-spatial positive topology of the form $(S, \triangleleft \mathcal{X}, \ltimes \mathcal{X})$, with $\mathcal{X}$ a concrete space, would be thick and hence it would coincide with the locale $\left(S, \triangleleft_{\mathcal{X}}, \triangleleft_{\triangleleft \mathcal{X}}\right)$. This would make weak sobriety coincide with sobriety. As for the other statement, note that if all locales were strongly overt, then they would all be overt, which is not the case intuitionistically.

\section{Basic topologies}

Sometimes it is useful to consider a structure more general than that of a positive topology, namely that of a basic topology, as introduced by the second author [21, 22]. This is a triple $(S, \triangleleft, \ltimes)$ where $\triangleleft$ is just a basic cover, that is, a relation which satisfies all but the last condition in the definition of a formal cover.

From an algebraic point of view, a basic cover is a presentation of a suplattices (complete join-semilattice), actually a set-based one. A suplattice homomorphism (a map which preserves all joins) between two set-based suplattices can be presented as a particular binary relation (up to a suitable equivalence) between the corresponding bases.

The definition of $\triangleleft \mathcal{X}$ in equation (1), if applied to the case when $\mathcal{X}=(X, \Vdash, S)$ is just a binary relation between two sets, produces a basic cover, in general. On the other hand, equation (2) does not depend on $\mathcal{X}$ being a concrete space, and so it produces a positivity relation $\ltimes \mathcal{X}$ even if $\Vdash$ is just a relation. So $(S, \triangleleft \mathcal{X}, \ltimes \mathcal{X})$ is a basic topology which we say to be representable by the relation $\mathcal{X}$.

Let $\operatorname{Closed}(\mathcal{S})$ be the suplattice of formal closed subsets of a basic topology $\mathcal{S}$ (joins are given by unions). 
Proposition 6.1 For every basic topology $\mathcal{S}$, the following are equivalent:

(1) $\mathcal{S}$ is representable by a relation (as a basic topology).

(2) $\mathcal{S}$ is strongly overt, and $\operatorname{Closed}(\mathcal{S})$ is set-based.

Proof If $\mathcal{S}=\mathcal{S}_{\mathcal{X}}$ for a certain $\mathcal{X}=(X, \Vdash, S)$, then $\diamond x$ is a formal closed set for every $x \in X$. So if $a \in V \Rightarrow U \curlyvee V$ for every formal closed $V$, then in particular $\forall x(a \in \diamond x \Rightarrow U \curlyvee \diamond x)$. This is $\forall x(x \Vdash a \Rightarrow(\exists u \in U)(x \Vdash u))$, that is, $a \triangleleft \mathcal{X} U$. This shows that $\triangleleft=\triangleleft_{\mathcal{X}}=\triangleleft_{\ltimes}$ and hence $\mathcal{S}$ is strongly overt. Now if $V$ is formal closed, then $a \in V$ if and only if $a \ltimes \mathcal{X} V$ if and only if there exists $x \in X$ such that $a \in \diamond x \subseteq V$. So $V$ is a union of subsets of the form $\diamond x$. This says that $\{\diamond x \mid x \in X\}$ is a base for $\operatorname{Closed}(\mathcal{S})$.

Conversely, assume that $\operatorname{Closed}(\mathcal{S})$ is set-based with base $\left\{V_{i} \mid i \in I\right\}$. Let $\mathcal{X}$ be $(I, \Vdash, S)$, where $i \Vdash a$ is $a \in V_{i}$, so that $\diamond i=V_{i}$ for every $i \in I$. So $\ltimes=\ltimes \mathcal{X}$ by an easy calculation. By strong overtness, $a \triangleleft U$ means that $a \in V$ implies $U \gamma V$ for every $V$ formal closed, which turns out to be equivalent to $a \in V_{i} \Rightarrow U \gamma V_{i}$ for every $i \in I$. Thus $a \triangleleft U$ is the same as $a \triangleleft \mathcal{X} U$.

Under an impredicative view, of course, this says that "strongly overt" and "representable by a relation" are one and the same notion.

Representable by a concrete space versus representable by a relation. A positive topology which is representable by a relation need not be representable by a concrete space, as we now show.

In a classical and impredicative framework, all locales are strongly overt and hence representable by a relation. However not all locales are representable by a concrete space, otherwise they would all be spatial.

Constructively, a similar counterexample can be given as follows. Put $S=\mathbb{Q} \times \mathbb{Q}$ where $\mathbb{Q}$ is the set of rational numbers. Think of $(a, b) \in S$ as an open interval on the real line. Consider the binary relation $(x, y) \gtrless(a, b)$ on $S$ defined by $\max \{x, a\}<\min \{y, b\}$, which says that the two intervals overlap. Consider next the basic topology $(S, \triangleleft, \ltimes)$ represented by $(S, \ngtr, S)$. For $a, b \in \mathbb{Q}$ and $U \subseteq S$, the statement $(a, b) \triangleleft U$ says that the interval $(a, b)$ is contained in the closure of the open set corresponding to $U$, namely the union of all intervals in $U$. This implies that the complete lattice of formal opens is isomorphic to that of regular open subsets of the real line (see, for instance, Ciraulo and Sambin [6]). In particular, such a lattice is a frame and hence $(S, \triangleleft, \ltimes)$ is a positive topology. Since the locale of regular open subsets of the real line has no points, 
a fortiori $(S, \triangleleft, \ltimes)$ has no ideal points. Therefore $(S, \triangleleft, \ltimes)$ is not spatial, hence not representable by a concrete space.

This counterexample also shows that a representable positive topology need not be spatial, nor reducible (otherwise it would be also spatial, since it is already strongly overt; recall Proposition 5.3). Consequently, a strongly overt positive topology need not be spatial, nor reducible.

\section{Acknowledgements}

We thank our colleagues Milly Maietti and Tatsuji Kawai for fruitful discussions.

\section{References}

[1] P Aczel, G Curi, On the $T_{1}$ axiom and other separation properties, Ann. Pure Appl. Logic 161 (2010) 560-569; https://doi.org/10.1016/j.apal.2009.03.005

[2] P Aczel, C Fox, Separation properties in constructive topology, from: "From sets and types to topology and analysis", (L Crosilla, P Schuster, editors), Oxford Logic Guides 48, Oxford Univ. Press, Oxford (2005) 176-192; https://doi.org/10.1093/acprof:oso/9780198566519.003.0011

[3] J Berger, H Ishihara, P Schuster, The Weak König Lemma, Brouwer's Fan Theorem, De Morgan's Law, and Dependent Choice, Rep. Math. Log. 47 (2012) 63-86

[4] F Ciraulo, ME Maietti, G Sambin, Convergence in formal topology: a unifying notion, J. Log. Anal. 5 (2013) Paper 2, 1-45; https://doi.org/10.4115/jla.2013.5.2

[5] F Ciraulo, G Sambin, Finitary formal topologies and Stone's representation theorem, Theoret. Comput. Sci. 405 (2008) 11-23; https://doi.org/10.1016/j.tcs.2008.06.020

[6] F Ciraulo, G Sambin, The overlap algebra of regular opens, J. Pure Appl. Algebra 214 (2010) 1988-1995; https://doi.org/10.1016/j.jpaa.2010.02.002

[7] F Ciraulo, G Sambin, A constructive Galois connection between closure and interior, J. Symbolic Logic 77 (2012) 1308-1324; https://doi.org/10.2178/jsl.7704150

[8] F Ciraulo, S Vickers, Positivity relations on a locale, Ann. Pure Appl. Logic 167 (2016) 806-819; https://doi.org/10.1016/j.apal.2016.04.009

[9] T Coquand, G Sambin, J Smith, S Valentini, Inductively generated formal topologies, Ann. Pure Appl. Logic 124 (2003) 71-106; https://doi.org/10.1016/S0168-0072(03)00052-6

[10] G Curi, On the collection of points of a formal space, Ann. Pure Appl. Logic 137 (2006) 126-146; https://doi.org/10.1016/j.apal.2005.05.019 
[11] M P Fourman, R J Grayson, Formal spaces, from: "The L. E. J. Brouwer Centenary Symposium (Noordwijkerhout, 1981)", Stud. Logic Found. Math. 110, North-Holland, Amsterdam (1982) 107-122; https://doi.org/10.1016/S0049-237X(09)70126-0

[12] M P Fourman, J M E Hyland, Sheaf models for analysis, from: "Applications of sheaves", (M P Fourman, D Scott, editors), Lecture Notes in Math. 753, Springer, Berlin (1979) 280-301

[13] C Fox, Point-set and point-free Topology in Constructive Set Theory, PhD thesis, University of Manchester (2005)

[14] N Gambino, P Schuster, Spatiality for formal topologies, Math. Structures Comput. Sci. 17 (2007) 65-80; https://doi.org/10.1017/S0960129506005810

[15] M E Maietti, A minimalist two-level foundation for constructive mathematics, Ann. Pure Appl. Logic 160 (2009) 319-354; https://doi.org/10.1016/j.apal.2009.01.006

[16] ME Maietti, Why developing formal topology in the minimalist foundation (2010) Slides of a talk given at Constructive Mathematics: Proofs and Computation, June 7-11, Fraueninsel (Chiemsee), Germany

[17] ME Maietti, G Sambin, Toward a minimalist foundation for constructive mathematics, from: "From sets and types to topology and analysis", (L Crosilla, P Schuster, editors), Oxford Logic Guides 48, Oxford Univ. Press, Oxford (2005) 91-114; https://doi.org/10.1093/acprof:oso/9780198566519.003.0006

[18] M E Maietti, G Sambin, Why topology in the minimalist foundation must be pointfree, Log. Log. Philos. 22 (2013) 167-199; https://doi.org/10.12775/LLP.2013.010

[19] D Rinaldi, G Sambin, P Schuster, The Basic Zariski Topology, Confluentes Mathematici 7 (2015) 55-81; https://doi.org/10.5802/cml.18

[20] G Sambin, Intuitionistic formal spaces - a first communication, from: "Mathematical logic and its applications (Druzhba, 1986)", Plenum, New York (1987) 187-204

[21] G Sambin, Some points in formal topology, Theoret. Comput. Sci. 305 (2003) 347-408; https://doi.org/10.1016/S0304-3975(02)00704-1

[22] G Sambin, Positive Topology via the Basic Picture. New structures emerging from constructive mathematics, Oxford Logic Guides, Oxford Univ. Press, Oxford (to appear)

[23] G Sambin, G Trentinaglia, On the meaning of positivity relations for regular formal spaces, J. Univers. Comput. Sci. 11 (2005) 2056-2062

[24] G Sambin, S Valentini, P Virgili, Constructive domain theory as a branch of intuitionistic pointfree topology, Theoret. Comput. Sci. 159 (1996) 319-341; https://doi.org/10.1016/0304-3975(95)00169-7

[25] A S Troelstra, D van Dalen, Constructivism in mathematics. An introduction. Vol. I, volume 121 of Studies in Logic and the Foundations of Mathematics, North-Holland Publishing Co., Amsterdam (1988)

[26] S Vickers, Sublocales in formal topology, Journal of Symbolic Logic 72 (2007) 463-482; https://doi.org/10.2178/jsl/1185803619 
Dipartimento di Matematica “Tullio Levi Civita”, Università degli Studi di Padova, Via Trieste 63, 35121 Padova, Italy

ciraulo@math.unipd.it, sambin@math.unipd.it

http://www.math.unipd.it/ ciraulo, http://www.math.unipd.it/ sambin

Received: 27 August $2018 \quad$ Revised: 17 May 2019 